\title{
Heart rate variability and cardiac autonomic function in men with chronic alcohol dependence
}

\author{
Simon C Malpas, Edwin A Whiteside, Timothy J B Maling
}

\begin{abstract}
Cardiac autonomic function was studied in 23 alcohol dependent men by standard tests of autonomic function and measurement of 24 hour heart rate variability. In all there was peripheral or central nervous system damage or both. Standard tests of autonomic function showed vagal neuropathy in seven. The remainder had normal autonomic function tests. Twenty four hour heart rate variability was measured as the standard deviation of the successive differences between $R R$ intervals from an ambulatory electrocardiogram recording. Twenty four hour heart rate variability was significantly lower in both alcohol dependent groups than in controls, but the results in the two alcohol dependent groups were not significantly different from each other. The results of standard tests of autonomic function did not distinguish between the alcohol dependent men with normal autonomic function and controls. The differences in heart rate variability between this group and the controls may have been the result of the ability of this method to detect small changes in autonomic integrity. Cardiomyopathy may also account for some of these differences and such abnormalities should be excluded before results are to be regarded as a reflection of vagal function. Twenty four hour measurement of heart rate variability may be a more useful index of cardiac vagal neuropathy than currently available tests of autonomic function.
\end{abstract}

Damage to the autonomic nervous system affecting sudomotor, vasomotor, and cardiac function is a recognised feature of chronic alcohol dependence. ${ }^{1}$ Duncan et al indicated that vagal neuropathy is a common finding associated with impairment of the central and peripheral nervous systems. ${ }^{2}$ Sympathetic dysfunction affecting the control of blood pressure is, however, rare, ${ }^{3}$ though orthostatic hypotension may occur in a few patients and is associated with severe autonomic degeneration. ${ }^{4}$

Commonly applied tests of cardiac autonomic function (Valsalva's manoeuvre, deep breathing, and standing) examine heart rate responses to various manoeuvres that change the length of the cardiac cycle (RR interval) over a short period. The change in the $R R$ interval is assumed to be due to autonomic activity but this does not take into account that inputs of different frequencies produce the change in heart rate; those at lower frequencies are not necessarily autonomic in origin such as reflex mechanisms involved with continuing haemodynamic changes. Tests of autonomic function also tend to classify patients merely on the basis of the presence of neuropathy and give little scope for grading its severity. ${ }^{5}$ Measurement of short term ( $5 \mathrm{~min}$ ) heart rate variability has been advocated as an indicator of autonomic neuropathy ${ }^{6}$; however, there is disagreement about whether the recording should be performed with the patient standing, sitting, or lying and about the best index for measuring heart rate variability. ${ }^{7}$ Ambulatory electrocardiographic (Holter) monitoring of heart rate variability may provide a more sensitive index of autonomic function than is currently available ${ }^{8}$ because it makes a large number of RR intervals available for the calculation of heart rate variability. Monitoring over 24 hours also provides an opportunity to examine autonomic function during normal daily activities without interference from investigators. It may enable disease progression in alcohol dependent patients to be monitored. Ewing and colleagues reported that heart rate variability was reduced in diabetic patients who had normal standard autonomic function tests. ${ }^{8}$ The present study was conducted to test whether similar results apply to alcohol dependent patients and whether 24 hour measurement of heart rate variability is a useful method for assessing cardiac autonomic changes in alcohol dependent patients.

\section{Patients and methods}

We assessed autonomic integrity by standard tests of autonomic function and measurement of 24 hour heart rate variability in 23 alcohol dependent men (aged 37-72 (mean 54)) who had been drinking between 100 and $350 \mathrm{~g}$ of ethanol daily for $10-40$ years. At the time of study all these men were undergoing rehabilitation at a Salvation Army hostel. They were not admitted to hospital for the study. They had abstained from alcohol for one to nine weeks (mean three). None of them had hypertension, diabetes, or clinical evidence of heart disease or were experiencing withdrawal symptoms at the time of study. An electrocardiogram was taken to exclude men with cardiac rhythm abnormalities. Men were selected on the basis of abnormalities of the peripheral and central nervous system by a 
previously described protocol from our laboratory. ${ }^{2}$ We also assessed 11 healthy men (aged 34-68). The protocol was approved by the Wellington Hospital Board Ethical Committee and all participants gave their informed consent before taking part.

STANDARD TESTS OF AUTONOMIC FUNCTION All subjects underwent the following tests of autonomic function in the order below. They lay supine for 20 minutes before the start of the tests. The methods are described elsewhere. ${ }^{2}$

Deep breathing-Sinus arrhythmia of $<15$ beat/min was regarded as abnormal.

Standing-The 30:15 ratio of the response of heart rate to standing was measured; a ratio $<1.03$ was defined as abnormal. The change in blood pressure upon standing was also measured, and a fall $>10 \mathrm{~mm} \mathrm{Hg}$ diastolic and $>20 \mathrm{~mm} \mathrm{Hg}$ systolic indicated sympathetic failure.

Valsalva manoeuvre-Subjects held a forced expiratory pressure of $40 \mathrm{~mm} \mathrm{Hg}$ for $15 \mathrm{~s}$. The Valsalva ratio of the smallest heart beat interval during straining to the longest after the release of pressure was calculated. A ratio $<1.21$ was defined as abnormal.

Neck suction-The carotid baroreceptors were stimulated by the application of suction $(-50$ $\mathrm{mm} \mathrm{Hg}$ ) to the neck. ${ }^{9}$ The suction was applied rapidly $(-600 \mathrm{~mm} \mathrm{Hg} / \mathrm{s})$ and held for $3 \mathrm{~s}$. The longest $R R$ interval during the suction was measured. Failure to prolong the RR interval by $>120 \mathrm{~ms}$ was defined as abnormal.

Abnormal responses to two or more tests of autonomic function indicated autonomic neuropathy. ${ }^{10}$

\section{MEASUREMENT OF 24 HOUR HEART RATE \\ VARIABILITY}

The men were fitted with a Holter monitor (Oxford Medilog, MR14) that recorded two channels of electrocardiogram on to a standard 60 minute cassette tape over a 24 hour period. They kept a self-recorded diary of the day's events such as meal times, exercise, and sleep. Tapes were played back through an analyser (Oxford Medilog, MA14) at 60 times the recorded speed. Fluctuations in the recording speed were adjusted by a phase-locked loop time signal. The QRS waveform was filtered, detected electronically, and a pulse was generated corresponding to each $R$ wave. False triggering of the $T$ wave was inhibited by generating a relatively long width $(250 \mathrm{~ms}$ real time) for the resultant pulse. The time between $R$ waves was counted by a purpose-built rate logging device (accuracy $\pm 5 \mu \mathrm{s}$ ) and the data were relayed through a parallel interface to a personal computer (IBM) where the RR intervals were added sequentially to a file. The file containing the $R R$ intervals was divided into sections of 30 minutes of real time $R R$ intervals so that up to 48 subfiles were produced for each 24 hour recording. To eliminate failure of $\mathrm{QRS}$ detection, intervals outside a preset range (300 to $1800 \mathrm{~ms}$ ) and those tapes with more than five extrasystoles per hour were discarded from further analysis.

\section{STATISTICAL ANALYSIS}

The alcohol dependent men were divided into two groups on the basis of the presence or absence of autonomic neuropathy as defined by the results of the standard tests of autonomic function. Heart rate variability was assessed from the standard deviation of the successive differences between RR intervals (SDSD) for each $30 \mathrm{~min}$ subfile in each individual by a commercial statistical package (SAS Institute, USA) on a Digital Microvax computer. Six controls repeated the ambulatory monitoring between 1-12 weeks later (mean 3 ). The reproducibility coefficient for the SDSD was 0.83 ; therefore the method was repeatable. ${ }^{11}$ Results from individuals were standardised to a starting time of $0900 \mathrm{~h}$. Two way analysis of variance was used to compare the results of $24 \mathrm{~h}$ heart rate variability in controls and the two alcohol dependent groups by a Bonferroni multiple comparisons procedure. The sample size studied had sufficient power, at the $5 \%$ level, to detect a change of $>15 \%$ in heart rate variability between groups. Results are presented as means (SEM). Statistical significance was achieved when $\mathrm{p}<0.05$.

\section{Results}

\section{STANDARD AUTONOMIC FUNCTION TESTS}

Sixteen alcohol dependent men (group 1) had normal standard autonomic function tests. Vagal neuropathy was detected in seven (group 2). Mean responses to standing, Valsalva's manoeuvre, deep breathing, and neck suction were significantly different in group 2 and controls but not between group 1 and controls (table 1). None of the men had sympathetic neuropathy (orthostatic hypotension). There was no difference in age or duration of alcohol dependency between the two groups; however, the degree of peripheral neuropathy, or central nervous system impairment, or both was significantly greater in group 2 .

\section{TWENTY FOUR HOUR HEART RATE VARIABILITY}

Examination of the diary cards indicated that the alcohol dependent men were generally no more sedentary than the controls and that their times of meals and sleep periods did not differ greatly. All groups showed considerable circadian variation in $R R$ intervals with the slowest heart rate at $0500 \mathrm{~h}$ in the controls and group 2 and at $0600 \mathrm{~h}$ in group 1 . There was a tendency for both alcohol dependent groups to have higher heart rates throughout the $24 \mathrm{~h}$ period $(p=0.067)$ than the normal group. The

Table 1 Autonomic function test results from 11 controls, 16 alcohol dependent men without vagal neuropathy (group 1) and seven alcohol dependent men with vagal neuropathy (group 2) (mean (SEM))

\begin{tabular}{|c|c|c|c|}
\hline & Controls & Group 1 & Group 2 \\
\hline $\begin{array}{l}\text { Standing ( } 30: 15 \\
\text { ratio) } \\
\text { Valsalva ratio } \\
\text { Deep breathing } \\
\text { (beats/min) } \\
\text { Bradycardia with } \\
\text { neck suction (ms) }\end{array}$ & $\begin{array}{l}1.12(0.02) \\
1.74(0.09) \\
20(2 \cdot 2) \\
222(46)\end{array}$ & $\begin{array}{l}1.08(0.03) \\
1.60(0.11) \\
23(4.4) \\
245(47)\end{array}$ & $\begin{array}{l}1.03(0.02) \\
1.28(0.06) \\
7.8(1.9) \\
42(14)^{\star}\end{array}$ \\
\hline
\end{tabular}

$\star_{p}<0.05$. 
Figure $1 \quad R R$ intervals and mean heart rate variability ( $S D S D$ ) over $24 \mathrm{~h}$ in 11 controls, 16 group 1 and seven group 2.

There was a significant difference in heart rate variability between the alcohol dependent groups and the controls.

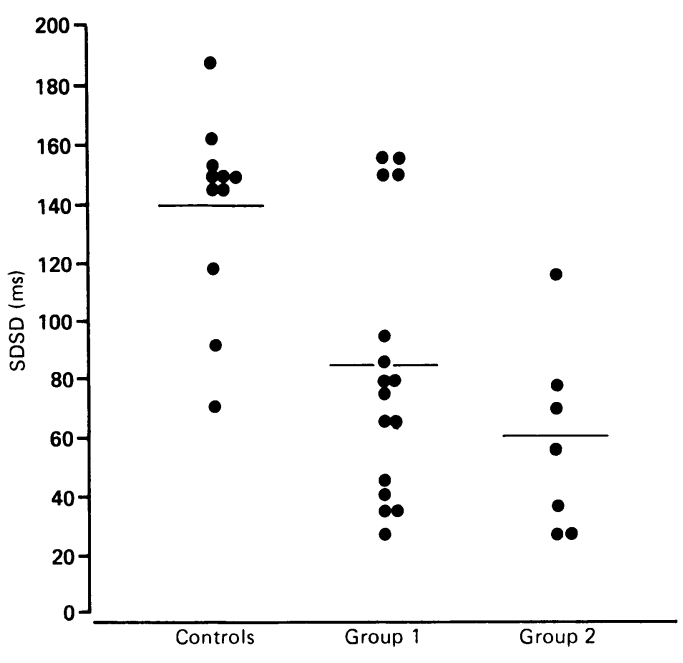

mean RR interval over $24 \mathrm{~h}$ was 806 (38) $\mathrm{ms}$ in the controls, 773 (29) $\mathrm{ms}$ in group 1, and 739 (23) $\mathrm{ms}$ in group 2.

Heart rate variability was significantly lower $(p<0.01)$ in both alcohol dependent groups than in the controls at all times over the $24 \mathrm{~h}$ period; however, the alcohol dependent groups were not significantly different from each other. The heart rate variability did not show the same degree of periodicity over $24 \mathrm{~h}$ as the
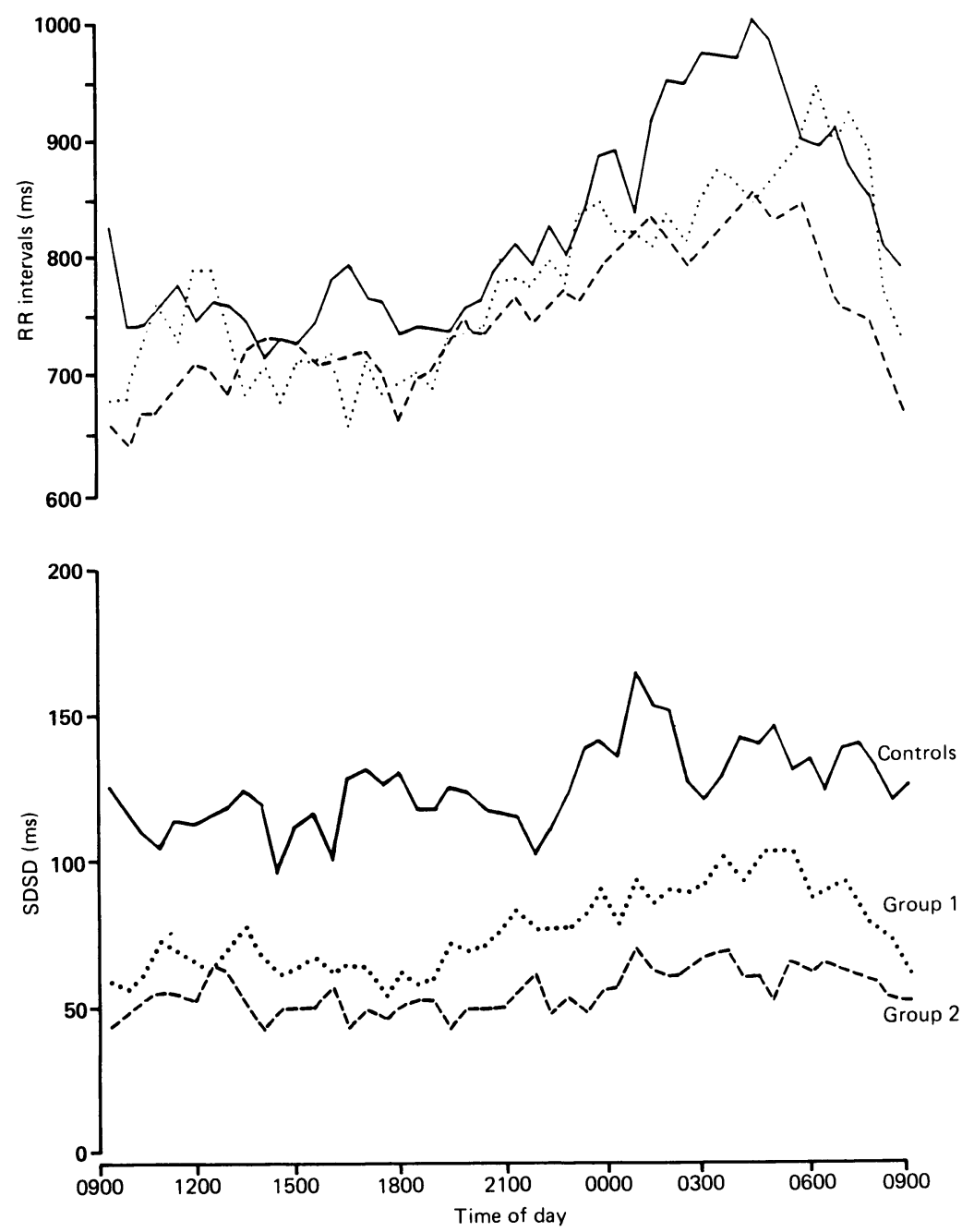

Figure 2 Individual mean heart rate variability (SDSD) in the three groups for the whole $24 \mathrm{~h}$ period. The solid line indicates the mean value in each group. There was $a$ significant difference between controls and both alcohol dependent groups that the standard tests of autonomic function did not identify.

Table 2 Mean heart rate variability ( $S D S D$ ) over six time periods in 11 controls, 16 alcohol dependent men without vagal neuropathy (group 1), and seven alcohol dependent men with vagal neuropathy (group 2) (mean (SEM))

\begin{tabular}{lrrr}
\hline & Controls & Group 1 & Group 2 \\
\hline Morning & & & \\
$\quad(0900-1130 \mathrm{~h})$ & $125(12)$ & $72(11)$ & $58(10)$ \\
Lunch $(1130-1400 \mathrm{~h}) 117(16)$ & $70(10)$ & $53(9)$ \\
$\begin{array}{l}\text { Afternoon } \\
\quad(1400-1730 \mathrm{~h})\end{array}$ & $126(13)$ & $68(9)$ & $50(10)$ \\
$\begin{array}{l}\text { Evening } \\
\quad(1700-0000 \mathrm{~h})\end{array}$ & $134(16)$ & $81(11)$ & $58(11)$ \\
$\begin{array}{l}\text { Sleep }(0000-0530 \mathrm{~h}) \\
\text { Waking }\end{array}$ & $152(17)$ & $100(17)$ & $71(20)$ \\
$\quad(0530-0900 \mathrm{~h})$ & $140(20)$ & $94(17)$ & $69(18)$ \\
\hline
\end{tabular}

$\mathrm{RR}$ intervals and no clear variation was apparent (fig 1). When the mean of the heart rate variability for morning, lunch, afternoon, evening, sleep, and waking was taken a tendency for variability to increase during sleep was seen (table 2). The increase during sleep was similar in all groups and only set at a lower point in alcohol dependent groups. The mean SDSD over $24 \mathrm{~h}$ was $138(10) \mathrm{ms}$ in the normal group, 83 (11) $\mathrm{ms}$ in group 1 , and 60 (12) $\mathrm{ms}$ in group 2 (fig 2 ). Twenty four hour SDSD values for 12 out of 16 of the group 1 men were well below the mean SDSD of the controls. Sixteen of the 23 alcohol dependent men had Valsalva ratios within the normal range, 10 had normal responses on standing, 12 for deep breathing, and nine in response to neck suction. Only six of the 23 alcohol dependent men had mean heart rate variability values within the normal range; this was significantly less than the proportion with normal tests of autonomic function.

\section{Discussion}

Heart rate variability was significantly reduced in alcohol dependent men with established vagal neuropathy and in some without. One explanation is that measurement of $24 \mathrm{~h}$ heart rate variability detected subtle changes in autonomic integrity in the men in whom standard tests of autonomic function were normal. The difference in the proportion of alcohol dependent men with heart rate variability or autonomic function test results within the normal range accords with this explanation and indicates that grouping alcohol dependent people according to the presence of autonomic neuropathy may hide important information. Our results were consistent with earlier evidence in diabetic patients ${ }^{8}$ that 24 hour monitoring of heart rate variability detected small changes in autonomic function which may not be detectable by standard autonomic function tests.

Alternatively the similarity between heart rate variability in the alcohol dependent men with normal autonomic function and with abnormal autonomic function may relate to disordered end organ function. Tests of autonomic function measure the end organ responses to reflex stimulation; therefore conclusions about the origin of abnormal responses are made from indirect measurements. In this 
study minor subclinical cardiomyopathy may have been present in a few men; this was not excluded by a chest $x$ ray or echocardiogram. Though Levin noted decreases in the Valsalva ratio in individuals with clinically overt cardiomyopathy ${ }^{12}$ the effect of subclinical cardiomyopathy on autonomic function has not been documented. The SDSD index of heart rate variability may be more affected by subclinical cardiomyopathy and the reduced heart rate variability in some alcohol dependent men with normal standard autonomic function tests may be the result of subclinical cardiomyopathy rather than vagal neuropathy. Previous studies of alcoholic vagal neuropathy did not exclude individuals with cardiomyopathy, ${ }^{1213}$ which is quite common in this group. ${ }^{14}$ It is unlikely, however, that cardiomyopathy accounts for all the reduction in heart rate variability in the alcohol dependent men with normal autonomic function tests, and some degree of vagal neuropathy is feasible. Because of these possibilities it may be inappropriate to apply autonomic function tests to alcohol dependent individuals. In future studies alcohol dependent individuals should be excluded if results are to be considered a reflection of vagal function.

In alcohol dependent individuals the risk of death was increased in those with vagal neuropathy. ${ }^{15}$ Most deaths in the study were sudden or unexplained. A reduced heart rate variability over 24 hours was associated with a 5.3 fold increase in the mortality in patients recovering after myocardial infarction. ${ }^{16}$ Furthermore heart rate variability was reduced in patients known to be at risk of sudden cardiac death ${ }^{17}$ The rise in heart rate variability during sleep that we found has been noted before. ${ }^{8}$ This may confer some extra stability on the sinoatrial axis because the arrhythmias decreased during orthodox slow wave sleep. ${ }^{18}$ It has been suggested that the integrity of the autonomic nervous system may be important in the prevention, promotion, or precipitation of cardiac arrhythmias and that reduced vagal efferent activity may favour cardiac electrical instability. ${ }^{19}$ Measurement of heart rate variability over 24 hours may be an appropriate means of assessing such alterations.

Twenty four hour measurement of heart rate variability is potentially an accurate test of autonomic function. This is gained by the addition of every RR interval occurring in 24 hours (up to 140000 beats per day) and through the measurement of heart rate variability by the SDSD which is unaffected by periods of activity. The SDSD has been used as a method for assessing short term heart rate variability in newborn infants ${ }^{20}$ and in individuals with multiple sclerosis and diabetes $^{21} 22$ but its application to 24 hour electrocardiographic recordings has been limited. ${ }^{8}$ Its sensitivity and statistical robustness when applied to ventricular extrasystoles has been critically examined. ${ }^{23}$ The SDSD acts as a statistical filter in which the low frequency components such as trends in heart rate caused by activity and neurohormonal effects are filtered out. Its interpretation therefore relies on the reproducibility of a stimulus, the cooperation of subjects, and the long term stability of the timing mechanisms in the recording equipment. Thus though the alcohol dependent groups had higher heart rates this would not account for the reduced heart rate variability. Neubauer and Gundersen proposed that the SDSD was the best method of detecting small and early abnormalities in autonomic function. $^{21}$ The SDSD produces a measure of beat to beat variability and is a composite of variability of all the higher frequencies present in the data that could be identified by other methods such as spectral analysis. ${ }^{24}$ This composite emphasises the higher frequencies because lower frequency variation due to blood pressure and thermoregulatory mechanisms usually causes minimal beat to beat changes that are not autonomic in origin. For these reasons the SDSD is likely to be a more accurate measure of autonomic function than the standard deviation of the RR intervals. ${ }^{16}$ It is also more appropriate for examining ambulatory autonomic function than spectral analysis because several constraints make spectral techniques unsuitable in examining all forms of data on the RR interval. ${ }^{25}$ The period of sleep is the most basal state of the day. Ambulatory monitoring provides an opportunity to examine cardiac autonomic function during this time. It is likely to be a reflection of natural background stimuli rather than the artificial manoeuvres conducted in laboratory based tests.

Tests of autonomic function have generally been developed and validated by application to patients with diabetes mellitus. There have been few examinations of heart rate variability in alcohol dependent patients ${ }^{13}$ and no previous long term recordings. Commonly used autonomic tests cause a temporary imbalance of the system examined, do not reflect its steady state function, and require active participation from the patient. These tests do not generally allow for the accurate detection of quantitative abnormalities in autonomic function beyond the description of abnormal or normal, though some attempts have been made to do so. ${ }^{5}$ Alcoholic polyneuropathy is generally accepted to be a result of nutritional factors rather than a neurotoxic effect of alcohol. ${ }^{26}$ The reported improvement in autonomic function with prolonged abstinence may be due to improved nutrition in some alcohol dependent patients. ${ }^{5}$ Provided cardiomyopathy is excluded, 24 hour monitoring may overcome many of the problems with conventional tests for measuring changes in cardiac autonomic function in alcohol dependent patients.

We thank Mr G Purdie for statistical advice and the Wellington Medical Research Foundation for financial support.

1 Melgaard B, Somnier F. Cardiac neuropathy in chronic

2 Duncan G, Johnson RH, Lambie DG, Whiteside EA. Evidence of vagal neuropathy in chronic alcoholics. Lancet Evidence of vagal

3 Di Marchi S, Cecchin E. Are orthostatic hypotension and impaired blood pressure control common features of the alcohol withdrawal syndrome? Clin Sci 1986;70:213-4.
Eisenhofer G, Whiteside EA, Johnson RH. Plasma cate- 
cholamine responses to change of posture in alcoholics during withdrawal and after continued abstinence from alcohol. Clin Sci 1985;68:71-8.

5 Tan ETH, Johnson RH, Lambie DG, Whiteside EA Alcoholic vagal neuropathy: recovery following prolonged abstinence. J Neurol Neurosurg Psychiatry 1984;47: 1335-7.

6 Wheeler T, Watkins PJ Cardiac denervation in diabetes. $B$ Med J 1973;iv:584-6.

7 Ewing DJ, Borsey DQ, Bellavere F, Clarke BF. Cardiac autonomic neuropathy in diabetes: comparison of measures of R-R interval variation. Diabetologia 1981 21:18-24.

8 Ewing DJ, Neilson JMM, Travis P. New method for assessing cardiac parasympathetic activity using 24 hou electrocardiograms. Br Heart J 1984;52:396-402

9 Eckberg DL, Cavanaugh MS, Mark AL, Abboud FM. A simplified neck suction device for activation of carotid baroreceptors. J Lab Clin Med 1975;85:167-73.

10 Hilsted J. Testing for autonomic neuropathy. Clin Res 1984:16:128-35.

11 Bartko J. The intraclass correlation coefficient as a measure of reliability. Psychol Rep 1966;19:3-11.

12 Levin AB. A simple test of cardiac function based upon the heart rate changes induced by the Valsalva's maneuver. heart rate changes induced

13 Weise F, Muller D, Krell D, Kielstein V, Koch RD. Hear rate variability of chronic alcoholics in withdrawal and abstinence. Clin Neurol Neurosurg 1985;87:95-8.

14 Steinberg JD, Hayden MT. Prevalence of clinically occult cardiomyopathy in chronic alcoholism. Am Heart J 1981 101:461-4

15 Johnson RH, Robinson BJ. Mortality in chronic alcoholics $J$ Neurol Neurosurg Psychiatry 1988;51:476-80.

16 Kleiger RE, Miller JP, Bigger JT, Moss AJ, and the Multicenter post-infarction research group. Decreased heart rate variability and its association with increased mortality after acute myocardial infarction. Am J Cardiol 1987;59:256-62.

17 Myers GA, Martin GJ, Magid NM, et al. Power spectral analysis of heart rate variability in sudden cardiac death: comparison to other methods. IEEE Trans Biomed Eng 1986;12:1149-56.

18 Pickering TG, Johnson J, Honour AJ. Comparison of the effects of sleep, exercise and autonomic drugs on ventricular extrasystoles, using ambulatory monitoring of electrocardiogram and electroencephalogram. Am J Med 1978;65:575-83.

19 Ben-David J, Zipes DP. Autonomic neural modulation of cardiac rhythm, Part I: Basic concepts. Modern Concepts of Cardiovascular Disease 1988;57:41-6.

20 Mazza NM, Epstein MAF, Haddad GG, Law HS, Mellins RB, Epstein RA. Relation of beat-to-beat variability to heart rate in normal sleeping infants. Pediatr Res 1980; 14:232-5.

21 Neubauer B, Gundersen HJG. Analysis of heart rate variations in patients with multiple sclerosis. J Neurol Neurosurg Psychiatry 1978;41:417-9.

22 Gundersen HJG, Neubauer B. A long term diabetic autonomic nervous abnormality. Diabetologia 1977;13:137-40.

23 Heslegrave RJ, Ogilive JC, Furedy JJ. Measuring baselinetreatment differences and heart rate variability: variance
trence versus successive difference meansquare and beats per minute interbeat intervals. Psychophysiology 1979;16: $151-7$

24 Akselrod S, Gordon D, Ubel FA, Shannon DC, Barger AC, Cohen RJ. Power spectrum analysis of heart rate fluctuation: a quantitative probe of beat-to-beat cardiovascular control. Science 1981;213:220-2.

25 Schechtman VL, Kluge KA, Harper RM. Time-domain system for assessing variation in heart rate. Med Biol Eng Comput 1988;26:367-73.

26 Mayer RF. Peripheral nerve function in vitamin B12 deficiency. Arch Neurol 1965;13:335-62. 